\title{
rBaltMIP, a recombinant alpha-type myotoxin inhibitor from Bothrops alternatus (Rhinocerophis alternatus) snake, as a potential candidate to complement the antivenom therapy
}

\author{
Norival A. Santos-Filho ${ }^{a, d, 1}$, Tiago S. Sousa ${ }^{a, 1}$, Johara Boldrini-França ${ }^{a}$,

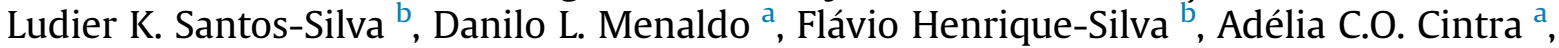 \\ Helen J. Laure ${ }^{\text {e }}$, Carla C.N. Mamede ${ }^{\mathrm{c}}$, Fábio Oliveira ${ }^{\mathrm{c}}$, Thalita B. Riul ${ }^{\mathrm{a}}$, \\ Marcelo Dias-Baruffi a , José C. Rosa ${ }^{\text {e }}$, Suely V. Sampaio ${ }^{\text {a, * }}$ \\ a Departamento de Análises Clínicas, Toxicológicas e Bromatológicas, Faculdade de Ciências Farmacêuticas de Ribeirão Preto, Universidade de São Paulo, \\ FCFRP-USP, Ribeirão Preto, SP, Brazil \\ ${ }^{\mathrm{b}}$ Departamento de Genética e Evolução, Universidade Federal de São Carlos, UFScar, São Carlos, SP, Brazil \\ ${ }^{\mathrm{c}}$ Departamento de Ciências Fisiológicas, Instituto de Ciências Biomédicas, Universidade Federal de Uberlândia, Uberlândia, MG, Brazil \\ ${ }^{\mathrm{d}}$ Departamento de Bioquímica e Tecnologia Química, Instituto de Química, Univ. Estadual Paulista, Araraquara, SP, Brazil \\ e Departamento de Biologia Celular e Molecular e Bioagentes Patogénicos, Faculdade de Medicina de Ribeirão Preto, Universidade de São Paulo, FMRP-USP, \\ Ribeirão Preto, SP, Brazil
}

\section{A R T I C L E I N F O}

\section{Article history:}

Received 1 August 2016

Received in revised form

24 October 2016

Accepted 27 October 2016

Available online 29 October 2016

\section{Keywords:}

Bothrops alternatus

rBaltMIP

Phospholipase $A_{2}$ inhibitor $\alpha$ PLI

\begin{abstract}
A B S T R A C T
Phospholipase $A_{2}$ inhibitors (PLIs) are important targets in the search and development of new drugs. This study aimed at evaluating the potential of an alpha-type phospholipase $\mathrm{A}_{2}$ inhibitor from Bothrops alternatus (Rhinocerophis alternatus) snake in its recombinant form (rBaltMIP) to complement the conventional antivenom therapy. Biochemical experiments showed that rBaltMIP presented pI 5.8 and molecular masses of $\sim 21 \mathrm{kDa}$ by SDS-PAGE and $19.57 \mathrm{kDa}$ by MALDI/TOF MS. After tryptic peptides sequencing, the results were compared with other PLIs available in databases, showing $100 \%$ identity between rBaltMIP and its native inhibitor BaltMIP and from 92\% to 96\% identity with other inhibitors. Myotoxic activities of BthTX-I and BthTX-II toxins were measured via plasma CK levels, showing myotoxic effective concentrations (EC50) of $0.1256 \mu \mathrm{g} / \mu \mathrm{L}$ and $0.6183 \mu \mathrm{g} / \mu \mathrm{L}$, respectively. rBaltMIP neutralized the myotoxicity caused by these two toxins up to $65 \%$, without promoting primary antibody response against itself. Nevertheless, this recombinant PLI was immunogenic when standard immunization protocol with Freud's adjuvant was used. In paw edema assays, EC50 of $0.02581 \mu \mathrm{g} / \mu \mathrm{L}$ and $0.02810 \mu \mathrm{g} / \mu \mathrm{L}$, respectively, were observed with edema reductions of up to $40 \%$ by rBaltMIP, suggesting its use as an additional antivenom. In addition, myotoxicity neutralization experiments with the myotoxin BthTX-I showed that rBaltMIP was more effective in inhibiting muscle damage than the conventional antivenom. Thus, considering the severity of envenomations due to Bothrops alternatus (Rhinocerophis alternatus) and the low neutralization of their local effects (such as myotoxicity) by the current antivenoms, rBaltMIP is a promising molecule for the development of novel therapeutic strategies for clinical applications.
\end{abstract}

(c) 2016 Elsevier Ltd. All rights reserved.

\section{Introduction}

Snakebites represent a social, economic and public health problem, causing serious debilitating consequences to the victims (Habib, 2013; Hifumi et al., 2015). Although the lethality induced by snake envenomations is low, the frequency of sequelae related to

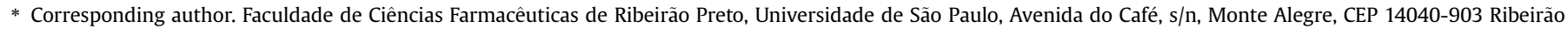
Preto, São Paulo, Brazil.

E-mail address: suvilela@usp.br (S.V. Sampaio).

1 These authors contributed equally in this article.
} 
local complication is higher. In 2009, the World Health Organization included snake envenomations in the list of neglected tropical diseases (Cruz et al., 2009; Harrison et al., 2009; Scheske et al., 2015; WHO, 2012). In Latin America (Gutiérrez, 2014) and in Brazil (Chippaux, 2015) envenomations still be a costly public health problem.

Nowadays, serum therapy using antisera composed of specific immunoglobulins is the only treatment for snake envenomations, but there are ongoing issues with availability, effectiveness and dosing (Scheske et al., 2015). These antivenoms neutralize the toxicity and lethality of specific venoms, but their administration is often associated with significant clinical side effects, such as serum sickness and renal failure (Morais and Massaldi, 2009; Zolfagharian and Dounighi, 2015). Additionally, the production of antivenoms is associated with high costs related to animal maintenance and also comes across animal welfare concerns, which instigates the search for innovative products for snakebite therapy (Krifi et al., 1999; Prado et al., 2016).

The search for natural inhibitors that neutralize snake venom toxins is of extreme importance for the production of more efficient antivenoms, especially considering that several toxins induce weak immunogenic responses, making traditional serum therapy unable to inhibit local effects of envenomations, such as the myotoxicity induced by phospholipases $\mathrm{A}_{2}\left(\mathrm{PLA}_{2} \mathrm{~S}\right)$ and $\mathrm{PLA}_{2}$-like enzymes (Kulkeaw et al., 2007; Santos-Filho et al., 2015a).

Venomous and non-venomous snakes present proteins in their blood, called phospholipase $A_{2}$ inhibitors (PLIs) (Marcussi et al., 2007; Oliveira et al., 2008; Santos-Filho et al., 2015a). These PLA 2 inhibitors are classified into three types: $\alpha, \beta$ and $\gamma$, according to their structural aspects, and they can neutralize several effects of snake venoms, including phospholipase, myotoxic, neurotoxic, inflammatory and edema-inducing activities (Lizano et al., 2003; Marcussi et al., 2007; Santos-Filho et al., 2015a).

To date, several $\alpha$ PLIs were purified from the blood of different snake species (Faure, 2000; Fortes-Dias, 2002; Lizano et al., 2003; Marcussi et al., 2007). One of these inhibitors is $\alpha$ BaltMIP, which was isolated from Bothrops alternatus (Rhinocerophis alternatus) snake plasma by Santos-Filho et al. (Santos-Filho et al., 2011). This inhibitor was characterized as a glycosylated oligomeric protein with subunits of $\mathrm{Mr}$ around 24,000, and had its $\mathrm{N}$-terminal sequence determined. $\alpha$ BaltMIP inhibitory activities were also assayed in relation to the toxic action of different PLA $A_{2}$ s, such as bothropstoxins I and II (BthTX-I and -II) from Bothrops jararacussu venom, piratoxins I and III (PrTX-I and -III) from B. pirajai venom, and crotoxin $\mathrm{B}(\mathrm{CB})$ from Crotalus durissus terrificus venom, showing potential to inhibit the anticoagulant, hemolytic, cytotoxic and myotoxic activities of the tested toxins.

Subsequently, Santos-Filho et al. (Santos-Filho et al., 2014) expressed a recombinant $\alpha$-type inhibitor from B. alternatus (Rhinocerophis alternatus) snake, called rBaltMIP, using a heterologous system in Pichia pastoris, also conducting structural, biochemical and functional characterization experiments. The expressed protein was tested regarding its ability to inhibit the phospholipase activity of different $\mathrm{PLA}_{2} \mathrm{~S}$ and was also effective in decreasing the myotoxic activity of different Asp49 (BthTX-II and PrTX-III) and Lys49 (BthTX-I and PrTX-I) enzymes. The inhibition of the myotoxic activity was also performed without prior incubation of myotoxins/ inhibitor. Furthermore, the cytotoxic activity of Asp49 PLA 2 S and Lys49 PLA $_{2}$-like enzymes was decreased after incubation with rBaltMIP.

Considering that conventional antivenoms are usually inefficient against the local effects induced by myotoxins after envenomations, the present study aims at investigating the possible use of rBaltMIP as a complement for the traditional serum therapy. Furthermore, in order to supplement the previous findings on this inhibitor, we also described further biochemical and functional characteristics and evaluated the immunogenic potential of rBaltMIP.

\section{Materials and methods}

\subsection{Animals}

Male Swiss (18-22 g) and BALB/c (18-22 g) mice were provided by the Animal Facilities of University of São Paulo, Campus Ribeirão Preto-SP. All procedures involving animals were approved by the Ethics Committee on Animal Use of University of São Paulo Campus Ribeirão Preto (CEUA no 13.1.276.53.1).

\subsection{Venom, antivenom and toxins}

Crude venom was acquired from the Center for Extraction of Animal Toxins (CETA) (Morungaba - SP, Brazil). Commercial polyvalent veterinary antivenom (AV) was purchased (Rasg \& Aplik; lema bioLOGIC do Brasil Ltda) and used in a molar ratio of $1: 1$ (rBaltMIP:BthTX-I). BthTX-I and II were isolated from B. jararacussu venom using Sephadex G-75 and CM-Sepharose columns as previously described (Cintra et al., 1993; Homsi-Brandeburgo et al., 1988), with slight modifications. The homogeneity of all isolated proteins was confirmed by SDS-PAGE and RP-HPLC.

\subsection{Expression and purification of rBaltMIP}

The induction of expression and purification of the recombinant inhibitor was accomplished as previously described by Santos-Filho et al. (Santos-Filho et al., 2014).

\subsection{Reverse phase chromatography}

In order to analyze the homogeneity of the toxins and the recombinant inhibitor, a reverse phase high performance liquid chromatography (RP-HPLC) was performed using a C18 column (250 $\times 4.6 \mathrm{~mm}$, CLC-ODS, Shimadzu, Japan $)$ previously equilibrated with solvent A [0.1\% trifluoroacetic acid (TFA)] and eluted with a concentration gradient of solvent B [70\% acetonitrile (ACN) and $0.1 \%$ TFA] at a flow rate of $1 \mathrm{~mL} / \mathrm{min}$. Chromatography was performed on a HPLC Shimadzu system and fractions were monitored at $280 \mathrm{~nm}$.

\subsection{Biochemical characterization}

Chromatography fractions, isolated toxins and the recombinant inhibitor were monitored by SDS-PAGE (Laemmli et al., 1970). Molecular masses were estimated by the interpolation of a linear logarithmic curve of relative molecular mass of standard proteins (14.4-116 kDa, unstained low range standard, Thermo Scientific) versus the distance of migration of the analyzed proteins in the gel. Isoelectric focusing of rBaltMIP was carried out according to Vesterberg (Vesterberg, 1972), with minor modifications. For protein quantification, sample solutions were analyzed using the microbiuret method (Itzhaki and Gill, 1964) or the method described by Scopes (Scopes, 1974).

\subsection{Molecular mass determination by mass spectrometry}

The molecular mass of rBaltMIP was determined in a MALDITOF/TOF mass spectrometer (AXIMA PERFORMANCE, Shimadzu Biotech - Launchpad 2.8.3 software; Kratos-Shimadzu, Manchester, UK), using sinapinic acid (10 mg/mL in $50 \%$ acetonitrile/ $0.1 \%$ TFA) as matrix at 1:1 ratio (sample:matrix). MS spectra were obtained in linear positive mode and the detected ions had their 
masses determined in relation to a calibration standard (Protein MALDI-MS Calibration Kit; Sigma, MSCAL-1-1 KT, 057k6815) containing the following proteins: insulin (bovine), cytochrome $\mathrm{C}$ (horse), aldolase (rabbit) and BSA (bovine serum albumin). The molecular mass range evaluated was of $5000-100,000 \mathrm{~m} / \mathrm{z}$.

\subsection{Amino acid sequencing of rBaltMIP tryptic peptides by tandem mass spectrometry}

\subsubsection{In situ tryptic hydrolysis}

rBaltMIP sample was diluted in $0.1 \mathrm{M}$ ammonium bicarbonate, reduced with $45 \mathrm{mM}$ DTT and alkylated with $100 \mathrm{mM}$ iodoacetamide (Sigma-Aldrich) prior to the addition of a trypsin solution $(0.5 \mu \mathrm{g}$ in $20 \mu \mathrm{L}$ of $0.1 \mathrm{M}$ ammonium bicarbonate) and incubation at $37{ }^{\circ} \mathrm{C}$ for $24 \mathrm{~h}$. The reaction was stopped by adding $5 \mu \mathrm{L}$ of formic acid. Thereafter, the tryptic peptides were desalted in a micro reversed phase column (POROS R2, Perceptive Biosystems, USA) previously activated with methanol and equilibrated with $0.2 \%$ formic acid. Samples were loaded onto the column and purified from salts and other hydrophilic components by 3 washes with $100 \mu \mathrm{L}$ of $0.2 \%$ formic acid, followed by elution of peptides with $30 \mu \mathrm{L}$ of a solution containing $60 \%$ methanol and $5 \%$ formic acid. Then, samples were dried in a vacuum centrifuge to be ready for mass spectrometry analysis.

\subsubsection{ESI Q-TOF}

The peptide samples were diluted with $20 \mu \mathrm{L}$ of a solution of $0.1 \%$ formic acid and $5 \%$ acetonitrile (ACN), being subjected to separation by UPLC-NanoAcquity (Waters, Beverly, MA) using a C18 reverse phase capillary column, directly coupled to the ionization source. The solutions used were $5 \% \mathrm{ACN}$ in $0.1 \%$ formic acid (A) and $95 \%$ ACN in $0.1 \%$ formic acid (B) at a flow rate of $300 \mathrm{~nL} / \mathrm{min}$ with an increasing linear gradient of solution B from $5 \%$ to $80 \%$ in $55 \mathrm{~min}$. Peptides were analyzed in an electrospray source coupled to a QTOF spectrometer (ESI-Q-TOF-MS, Ultima, Waters), using a voltage of $3.5 \mathrm{kV}$ for the ionization source, a cone voltage of $40 \mathrm{~V}$ and a source temperature of $100{ }^{\circ} \mathrm{C}$. The spectra were collected in datadependent-acquisition mode, and processed by the MassLynx 4.1 software. Collision-induced dissociation (CID-MS/MS) was performed automatically, selecting the most intense top 3 ions for a given time of chromatography. The peak list was generated by MassLynx software and submitted to Mascot server 2.4.1.

\subsubsection{Identification of peptides}

ESI Q-TOF spectra were processed and submitted to a database for identification of proteins using the Mascot software (version 2.4.1) against the NCBI database (NCBInr_20140323. fasta) following the parameters: no taxonomy, trypsin enzyme with loss of 1 cleavage (missed cleavage) and variable modifications to methionine oxidation and carbamidomethylation of Cys. Precursor ion tolerance was set to $1.2 \mathrm{Da}$ and MS/MS fragments to $0.8 \mathrm{Da}$. False-positive identifications (FDR) was set to $1 \%$, the mass spectra were submitted to the database in reverse mode, with a level of statistical significance of $\mathrm{p}<0.05$ corresponding to a permissible error of $5 \%$.

\subsection{Myotoxic activity}

Myotoxic activity was performed by measuring the levels of creatine kinase (CK) in the plasma of male BALB/c mice (18-22 g). Animals (groups $n=3$ ) were injected into the gastrocnemius muscle with $50 \mu \mathrm{L}$ of phosphate-buffered saline (PBS) alone (negative control) or containing different concentrations of Lys49 and Asp49 myotoxins (BthTX-I and II from B. jararacussu). After $3 \mathrm{~h}$ of injection, mice tail tips were cut and the blood was collected on heparinized capillary, which were immediately centrifuged at $3000 \times \mathrm{g}$ for $10 \mathrm{~min}$. The activity of the enzyme creatine kinase was determined using $4 \mu \mathrm{L}$ of plasma and $1 \mathrm{~mL}$ of the reagent from a CKUV K010 kinetic kit (Bioclin), determining the absorbance at $340 \mathrm{~nm}$ after $3 \mathrm{~min}$ of reaction at $37{ }^{\circ} \mathrm{C}$. The $\mathrm{CK}$ activity was expressed in units/L, with one unit representing the phosphorylation of one nmol of creatine per minute.

\subsubsection{Determination of BthTX-I and II's EC50 for myotoxicity}

The half maximal effective concentrations (EC50) of the toxins BthTX-I and II were calculated by a dose versus response curve. Animal groups $(\mathrm{n}=3)$ received samples containing different concentrations of the myotoxins $(0.05 ; 0.1 ; 0.2 ; 0.4 ; 0.8$ and $1.6 \mu \mathrm{g} / \mu \mathrm{L})$ in $50 \mu \mathrm{L}$ of PBS (the higher concentration was used only for BthTXII). The control group was injected with $50 \mu \mathrm{L}$ of PBS. After $3 \mathrm{~h}$, blood samples were collected and the plasma CK activity was determined as described in section 2.8. Three independent experiments were performed using triplicates for each toxin concentration. Graphs were plotted using the GraphPad Prism 5 software.

\subsection{Edema-inducing activity}

Male Swiss mice (18-22 g, $\mathrm{n}=3$ ) had their right paws measured with a low pressure caliper (Mitutoyo) and then received subplantar injection of $50 \mu \mathrm{L}$ of PBS (control group) or different concentrations of toxins (BthTX-I and II) solubilized in $50 \mu \mathrm{L}$ of PBS. After $30 \mathrm{~min}$, the paw size was measured again and the percentage of edema was calculated by the increased paw size compared to the initial measurements (before injection). Three independent experiments were carried out in triplicate for each concentration of both toxins.

\subsubsection{Determination of BthTX-I and II's EC50 for edema}

EC50 for the edema-inducing activity of BthTX-I and II were calculated by a dose versus response curve. For that, animals were subdivided in groups $(\mathrm{n}=3)$, with the control receiving only PBS and the others receiving different concentrations of the toxins $(0.0078 ; 0.0156 ; 0.0312 ; 0.0625 ; 0.125 ; 0.25 ; 0.5 ; 1.0$ and $2.0 \mu \mathrm{g} / \mu \mathrm{L})$.

\subsection{Neutralization of myotoxicity and edema by rBaltMIP}

These experiments were carried out as described in sections 2.8 and 2.9. The molar ratios of toxin/inhibitor were calculated using the EC50 values obtained for each toxin (2xEC50). The molar ratios used were $1: 1$ and $1: 2$ (toxin/inhibitor), with preincubation for $30 \mathrm{~min}$ at $37^{\circ} \mathrm{C}$ prior to administration in animals. In addition, we evaluated the neutralization of the myotoxic activity induced by B. jararacussu crude venom and BthTX-I using a commercial veterinarian snake antivenom (AV) (Rasg \& Aplik), alone or supplemented with rBaltMIP. Three independent experiments were performed in triplicate for each concentration of toxin (BthTX-I and II) and each assay.

\subsection{Histopathology of muscle fibers}

After collecting blood for the assessment of plasma CK levels, mice were euthanized by carbon dioxide inhalation followed by cervical dislocation. A section of the central region of their gastrocnemius muscle was then removed and soaked in fixation solution ( $10 \%$ formaldehyde in PBS, v/v). The material was dehydrated using increasing concentrations of ethanol and subsequently embedded in paraffin. The resulting blocks were cut into thick sections of $2.5 \mu \mathrm{m}$, stained with hematoxylin-eosin $(0.25 \% \mathrm{w} /$ v) and examined under an optical microscope (Santos-Filho et al., 2008, 2014). 


\subsection{Characterization of the immunogenic potential of rBaltMIP}

\subsubsection{Immunization of animals}

Initially, three female BALB/c mice (18-22 g) were anesthetized and blood samples $(200 \mu \mathrm{L})$ were collected by puncture of the orbital plexus to obtain pre-immune serum (negative control). Subsequently, mice were immunized via the intraperitoneal route with $10 \mu \mathrm{g}$ of antigen (rBaltMIP) diluted in $150 \mu \mathrm{L}$ of sterile PBS and emulsified with $150 \mu \mathrm{L}$ of complete Freund's adjuvant (CFA, Difco Laboratories Inc., Detroit, MI, USA). Fifteen days later, a second immunization was performed using incomplete Freud's adjuvant (IFA) instead of CFA. After another 15 days, a blood sample $(200 \mu \mathrm{L})$ was collected by puncture of the orbital plexus. After additional 15 days, mice were anesthetized for complete blood collection by cardiac puncture. Serum was separated from clots by centrifugation at $1500 \times \mathrm{g}$ for $10 \mathrm{~min}$, aliquoted and stored at $-20^{\circ} \mathrm{C}$. Afterwards, all sera samples were diluted (1:1000 to $1: 8000$ in PBS) and analyzed by ELISA. This immunization procedure was developed based on the protocols described by Harlow and Lane (Harlow and Lane, 1988).

Elicitation of anti-rBaltMIP antibodies was also evaluated in the experiments of neutralization of myotoxicity (section 2.10), using the inhibitor rBaltMIP $(50 \mu \mathrm{g})$ in the presence or absence of BthTX-I $(50 \mu \mathrm{g})$ and without CFA or IFA. Four groups of BALB/c mice $(\mathrm{n}=4)$ received intramuscular injections $(50 \mu \mathrm{L})$ of PBS (vehicle control), BthTX-I, rBaltMIP or BthTX-I plus rBaltMIP (previously incubated for $30 \mathrm{~min}$ at $37^{\circ} \mathrm{C}$ ). After 15 days, mice were anesthetized for complete blood collection by cardiac puncture, obtaining serum as mentioned above. Sera samples from each experimental group were analyzed by ELISA in the dilution of 1:4000.

\subsubsection{Detection of anti-rBaltMIP antibodies by ELISA}

The efficiency of animal immunization with rBaltMIP was monitored by ELISA. Initially, rBaltMIP was dissolved in PBS $(200 \mu \mathrm{g} / \mathrm{mL})$ and $50 \mu \mathrm{L}$ of this protein solution was loaded into the wells $(1 \mu \mathrm{g}$ per well) of a 96 wells polystyrene microtiter plates, followed by overnight incubation at $4{ }^{\circ} \mathrm{C}$ (antigen immobilization). Sequentially, each well was loaded with blocking buffer ( $3 \%$ gelatin, $0.5 \%$ Tween 20 in PBS, $200 \mu \mathrm{L}$ per well) and incubated for $2 \mathrm{~h}$ at $37{ }^{\circ} \mathrm{C}$ (blocking step). After this period, samples of preimmune- or immune-sera (50 $\mu \mathrm{L} /$ well), diluted 1:1000, 1:2000, 1:4000 and $1: 8000$ in PBS, were loaded to the plate and incubated for $1 \mathrm{~h}$ at $37^{\circ} \mathrm{C}$ (primary antibody incubation). Then, wells were loaded with $50 \mu \mathrm{L}$ of horseradish peroxidase conjugated anti-mouse IgG rabbit antibody (Jackson Immunoresearch, USA) diluted 1:5000 in PBS followed by incubation for $1 \mathrm{~h}$ at $37^{\circ} \mathrm{C}$ (secondary antibody incubation). All of the above steps were followed by washing with PBS containing $0.1 \%$ Tween $(200 \mu \mathrm{L}$ per well). Sequentially, TMB solution (tetramethylbenzidine, Organon Teknika, Boxtel, NL) in the presence of hydrogen peroxide was loaded into each well. Finally, after $10 \mathrm{~min}$, this reaction was stopped by adding $50 \mu \mathrm{L}$ of $2 \mathrm{~N}$ sulfuric acid solution per well, followed by reading the absorbance at $450 \mathrm{~nm}$ in a spectrophotometer (Spectramax Plus - Molecular Devices).

\subsection{Statistical analyses}

The results were expressed as means \pm standard deviation (SD). The significance of differences was determined by One-way ANOVA followed by Tukey post-test, with $\mathrm{p}<0.05$ considered significant. All the analyses were performed using the GraphPad Prism 5 program.

\section{Results and discussion}

Adequate treatments for snakebite envenomations are critically dependent on the ability of antivenoms to reverse venom-induced coagulopathy, myotoxicity, hypotensive shock and other effects (Fusco et al., 2015). It is known that, although antivenom therapy is effective against systemic toxic effects of envenomations, the conventional antivenoms present several limitations, such as the low efficacy against local effects induced by $\mathrm{PLA}_{2}$ and $\mathrm{PLA}_{2}$-like myotoxins, poorly neutralizing these toxins mainly due to the discrepancies between the pharmacokinetic profiles of low molecular mass toxins and antivenoms (Gutiérrez et al., 2003; Prado et al., 2016). Thus, improvements in the conventional serum therapy could be made with molecules such as PLIs that would be able to neutralize the local damages induced by snake toxins, without inducing toxic or immunogenic effects when administered in snakebite victims.

Although many studies have been investing in the characterization of natural inhibitors of snake toxins, such researches have to face challenges such as the necessity of finding new inhibitors (from plants, mammals, snakes, etc), the low availability and the difficulties in obtaining such inhibitors in sufficient quantities to allow functional, structural and biochemical studies. In the case of snakes, for example, obtaining PLIs from their blood come across ethical and environmental issues, since the animals need to be sacrificed in order to get these materials, and yet the amount of PLIs obtained is often insufficient for their full characterization. Thus, the heterologous expression of PLIs by molecular biology techniques greatly contributes to their studies, allowing their obtainment in larger quantities independently of snake serum.

In our previous studies, the heterologous expression of rBaltMIP was successfully achieved, making possible to obtain the purified recombinant inhibitor in larger quantities when compared to the native protein ( $\alpha$ BaltMIP) and in its active form, as shown by its capacity to partially reduce the PLA 2 and cytotoxic activities of both Asp49 and Lys49 enzymes and their in vivo myotoxicity (SantosFilho et al., 2014). Nevertheless, some important biological and structural questions remained unanswered, such as: Would rBaltMIP be efficient in complementing conventional antivenoms? Would it diminish the myotoxic effects of snake venoms? Would it cause antibody responses by itself?

Considering this, the present study aimed to further characterize rBaltMIP. Using the same protocol described in our previous work (Santos-Filho et al., 2014), we obtained the recombinant inhibitor and described some new biochemical and biological features of this molecule. rBaltMIP presented molecular mass of $\sim 21 \mathrm{kDa}$ by SDS-PAGE and 19.5 kDa by MALDI/TOF MS and pI of 5.8 (data not shown), demonstrating an acidic character like other aPLIs described in the literature, such as BaMIP from B. asper and aBjussuMIP from B. jararacussu snake (Lizano et al., 1997; Oliveira et al., 2008). Additionally, tryptic digestion of rBaltMIP resulted in 7 peptides (Table 1) that showed $100 \%$ sequence identity to the native inhibitor $\alpha$ BaltMIP previously described by Santos-Filho et al. (Santos-Filho et al., 2011), confirming the efficiency of the heterologous expression described by Santos-Filho et al (SantosFilho et al., 2014). and used in the present study to obtain the recombinant inhibitor.

It has been reported that $\alpha$ PLIs inhibit a variety of PLA 2 activities, such as enzymatic, myotoxic, cytotoxic, edema-inducing, among others (Lizano et al., 2003; Marcussi et al., 2007). Prior to the inhibition tests involving rBaltMIP and B. jararacussu myotoxins, we determined the effective concentrations (EC50) for the induction of myotoxicity and edema by BthTX-I $(0.1256 \mu \mathrm{g} / \mu \mathrm{L}$ and $0.02581 \mu \mathrm{g} /$ $\mu \mathrm{L}$, respectively) and BthTX-II $(0.6183 \mu \mathrm{g} / \mu \mathrm{L}$ and $0.02810 \mu \mathrm{g} / \mu \mathrm{L}$, respectively) (data not shown). These values were used to evaluate 
Table 1

rBaltMIP tryptic peptides determined by LC-ESI-Q-TOF mass spectrometry.

\begin{tabular}{|c|c|c|c|c|c|c|c|}
\hline Peptide & $\mathrm{m} / \mathrm{z}$ & Mr exp & Charge & Mr calc & Pep_delta & Pep_score & Pep_seq \\
\hline 1 & 449,75 & 897,48 & 2 & 897,50 & $-0,0168$ & 31,65 & GAFLTVYK \\
\hline 2 & 563,33 & 1124,64 & 2 & 1124,63 & 0,0012 & 33,85 & GAFLTVYKAR \\
\hline 3 & 460,25 & 918,48 & 2 & 918,49 & $-0,0144$ & 43,34 & AFANVLER \\
\hline 4 & 560,31 & 1118,61 & 2 & 1118,61 & 0,0059 & 37,43 & EIKNFEALR \\
\hline 5 & 818,96 & 1635,91 & 2 & 1635,91 & 0,0008 & 80,53 & LLNSLIDALMHLQR \\
\hline 6 & 750,03 & 2247,06 & 3 & 2247,09 & 0,0326 & 42,58 & QICEKADGHIPSPQLENQNK \\
\hline 7 & 1177,08 & 2352,15 & 2 & 2352,10 & 0,0434 & 99,13 & EAYLVVGDSANFTNWAAGEPNK \\
\hline
\end{tabular}

*Data obtained using Mascot program v.2.4.1 and NCBInr databank.

the inhibitory potential of rBaltMIP, using two toxin:inhibitor molar ratios (1:1 and $1: 2$ for myotoxicity, and $1: 2$ and $1: 7$ for edema) and considering the total activity of each myotoxin alone as $100 \%$.

Regarding the myotoxicity experiments, control groups that received only PBS showed basal CK levels (around 6\%) (Fig. 1A and B). BthTX-I in the presence of rBaltMIP at 1:1 and 1:2 M ratios showed significantly lower myotoxic activities, with inhibitions around 53 and 62\%, respectively (Fig. 1A), while for BthTX-II, the inhibition levels were of 55 and 57\%, respectively (Fig. 1B). The inhibitor by itself did not induce myotoxicity in comparison to the control group (Fig. $1 \mathrm{~A}$ and $\mathrm{B}$ ).

As discussed in our previous work (Santos-Filho et al., 2011), some authors pointed the C-terminal region as the main responsible for the toxic effects of Lys49 PLA 2 (Lomonte et al., 2003a, 2003b; Núñez et al., 2001; Santos-Filho et al., 2015b). However, the existence of other motifs that can participate or complement the action of the toxic effector site should not be discarded (Lomonte et al., 2003b). However, C-terminal peptides of Asp49 $\mathrm{PLA}_{2} \mathrm{~S}$ present no direct membrane damage activity, suggesting that the toxic mechanisms exerted by these proteins involves different pathways from that used by myotoxic Lys49 PLA $_{2}$ s, probably inducing muscle cell damage by affecting the integrity of plasma membranes, thereby leading to hyper contraction and other intracellular effects (Fuly et al., 2003; Núñez et al., 2001; SantosFilho et al., 2008).

In the edema-inducing experiments, PBS control groups showed an increase in paw thickness around 30\% in comparison to the initial measurements (Fig. 1C and D). When BthTX-I was injected along rBaltMIP at $1: 2$ and $1: 7 \mathrm{M}$ ratios, inhibitions of 17 and $20 \%$, respectively, were observed (Fig. 1C), while the inhibitions for BthTX-II were of 20 and 40\%, respectively (Fig. 1D). The inhibitor by itself did not induce edema in comparison to the control group (Fig. 1C and D).

Edema formation is the result of a synergism between various inflammatory mediators that increase the vascular permeability or the blood flow (Landucci et al., 2012). Other studies have also shown the ability of $\alpha$ PLIs to inhibit edema and myotoxic activities induced by different $\mathrm{PLA}_{2} \mathrm{~S}$ (Asp49 $\mathrm{PLA}_{2} \mathrm{~S}$ ) and PLA $\mathrm{P}_{2}$-like enzymes (Lys49 PLA ${ }_{2}$ S) (Lizano et al., 2000, 2003; Thwin et al., 2000). Our results showed that rBaltMIP had similar inhibitory effects on the myotoxicity induced by both BthTX-I and II, but was more efficient in inhibiting the edema induced by BthTX-II, an Asp49 PLA 2 , than that induced by BthTX-I, a Lys49 PLA 2 . This is somehow different from what was described for other $\alpha$ PLIs, such as $\alpha$ BjussuMIP from $B$. jararacussu snake plasma, which showed higher inhibitory effects on the edema induced by Lys 49 molecules in comparison to the Asp49 ones (Oliveira et al., 2008). BmjMIP, an $\alpha$ PLI from B. moojeni, also presented higher inhibitory potential on Lys49 $\mathrm{PLA}_{2} \mathrm{~S}$ (BthTX-I, PrTX-I, MjTX-I and II) in comparison to Asp49 $\mathrm{PLA}_{2} \mathrm{~S}$ (PrTX-III and BthTX-II) (Soares et al., 2003). BaMIP from B. asper also inhibited the edema-inducing activity of both Asp49 and Lys49 $\mathrm{PLA}_{2} \mathrm{~S}$, with higher inhibition of the latter (Lizano et al., 1997).
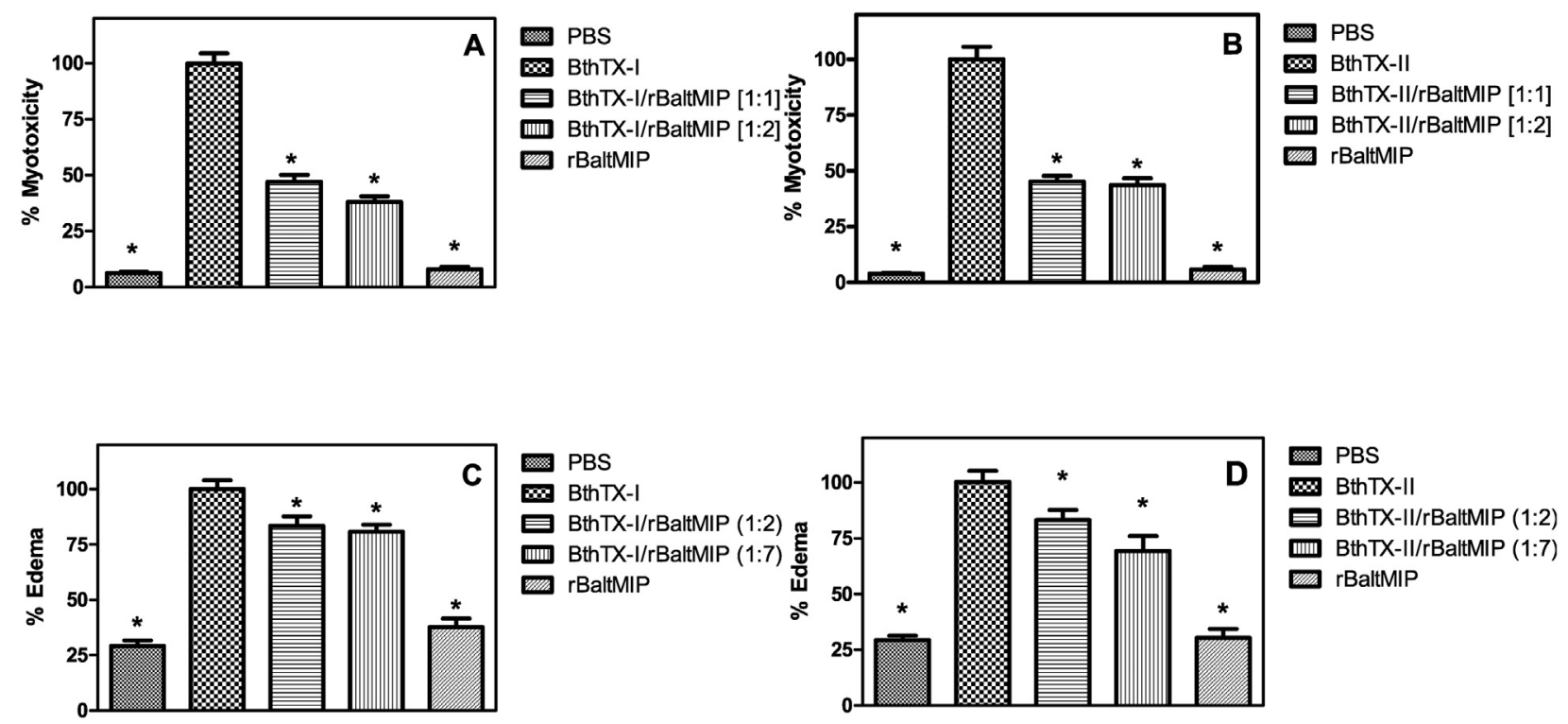

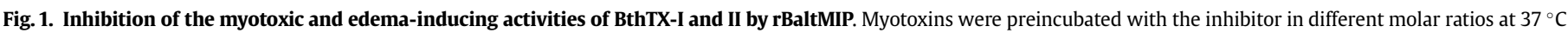

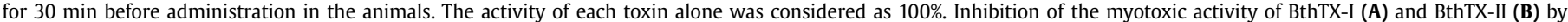

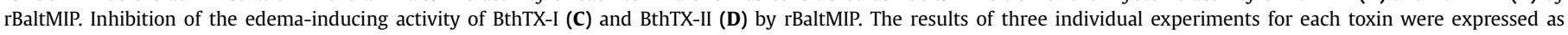
means \pm S.D. $(n=3)$ and statistically significant differences $(\mathrm{p}<0.05)$ were marked with $\left({ }^{*}\right)$. 
According to several works, the main structural domain responsible for the toxic effects of Lys49 PLA $_{2}$-like enzymes is the Cterminal region between the amino acid residues 115-129 (Lomonte et al., 2010). However, the mechanism of toxicity exerted by Asp49 myotoxins probably involves damage by affecting the integrity of the plasma membranes (Fuly et al., 2003; Santos-Filho et al., 2008). On the other hand, the probable mechanisms for edema induction by catalytically active Asp49 $\mathrm{PLA}_{2} \mathrm{~S}$ are related to the inflammatory reaction that takes place due to the hydrolysis of membrane phospholipids through the release of arachidonic acid or through activation of intracellular processes in target cells (Zuliani et al., 2005). Corroborating this hypothesis, studies have shown that chemical modification of His48 residue in the catalytic site by p-bromophenacyl bromide (BPB) induces loss of enzymatic activity and toxicity of snake venom $\mathrm{PLA}_{2} \mathrm{~S}$ (Santos-Filho et al., 2008; Soares and Giglio, 2003; Soares et al., 2004). However, the association between PLA $\mathrm{P}_{2}$ enzymatic activity and edema formation is complex, once that enzymatically inactive Lys49 PLA $_{2}$-like also elicits edema-forming activity, indicating that $\mathrm{PLA}_{2}$ could have pharmacological domain independent of the catalytic site (Bonfim et al., 2009; Kini and Evans, 1989; Nunes et al., 2011), which can interact with cellular membranes to induce cellular activation leading to a cytokine-dependent inflammatory reaction (Zuliani et al., 2005). As could be observed, the mechanisms by which $\mathrm{PLA}_{2} \mathrm{~S}$ induce paw edema are not clear and further studies are needed to investigate it (Bonfim et al., 2009).

Another interesting result is that rBaltMIP was more able to inhibit the myotoxic than edema-inducing activity. These results endorse the hypothesis that PLA $\mathrm{A}_{2} \mathrm{~S}$ could induce edema and myotoxicity by different mechanisms. Probably, rBaltMIP binds to myotoxic domains of myotoxins, avoiding toxicity; however, following this same hypothesis, rBaltMIP is not able to efficiently prevent edema because it does not bind to possible edema pharmacological sites.

It is important to cite that, the mechanism of inhibition of $\alpha$ PLIs is not totally clear. Several research groups hypothesized that the interaction between the inhibitor and proteins seems to occur in different ways (Estevão-Costa et al., 2016; Nishida et al., 2010; Okumura et al., 2005; Quirós et al., 2007; Santos-Filho et al., 2011, 2014). In a recent study, Estevão-Costa and co-workers (EstevãoCosta et al., 2016) suggested that the central pore, which is composed by positive charged residues, especially R57, K71, R108 and $\mathrm{H} 109$, could be a significant part of the binding site of $\alpha$ PLIs to acidic PLA $\mathrm{A}_{2} \mathrm{~S}$. However, the positive surface of the basic PLA $\mathrm{S}$ could prevent the PLA $/ \mathrm{PLI}$ interaction at the central pore, and according to these authors, the mechanism of inhibition of basic $\mathrm{PLA}_{2} \mathrm{~S}$ by aPLIs remains to be understood.

The main focus of our study was to evaluate rBaltMIP as a potential complement of the conventional serum therapy usually applied to treat snake envenomations. For that purpose, a polyvalent veterinary antivenom was purchased to be evaluated in addition to the recombinant inhibitor against the toxic effects of BthTX-I and B. jararacussu venom. The commercial antivenom was able to neutralize around $30 \%$ of the myotoxic activity of B. jararacussu venom (Fig. 2A) and BthTX-I (Fig. 2B), whereas rBaltMIP inhibited approximately $50 \%$ of the myotoxicity of the venom (Fig. 2A) and 60\% of that induced by BthTX-I (Fig. 2B). When the antivenom was complemented with rBaltMIP, an inhibition of around $60 \%$ was observed for both samples tested. The veterinary antivenom and rBaltMIP were also evaluated alone and did not
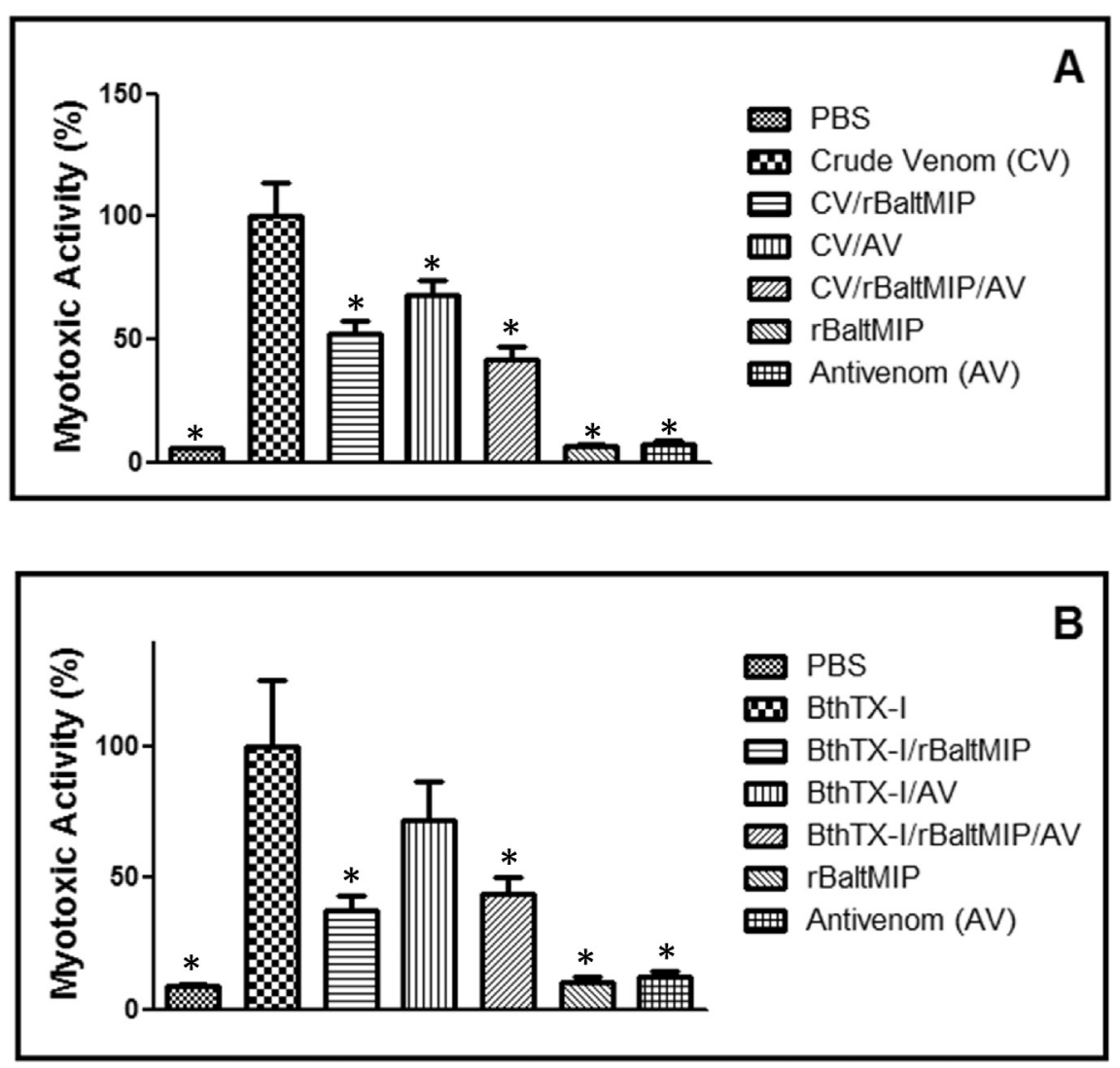

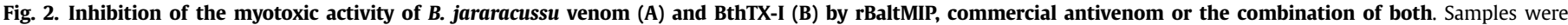

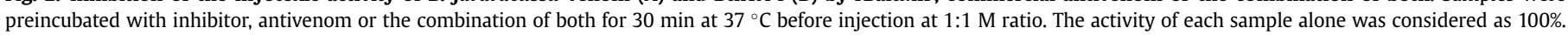
Results were expressed as means \pm S.D. $(n=3)$ and statistically significant differences $(\mathrm{p}<0.05)$ were marked with $\left({ }^{*}\right)$. 
induce myotoxicity on animals (Fig. 2).

Histopathological analysis showed that BthTX-I and B. jararacussu crude venom are capable of inducing damage to muscle fibers in animals, recruiting leukocyte infiltrates and forming an intense accumulation of intercellular fluid (Fig. 3A and B). In case of the crude venom, an intense hemorrhage was also induced, as illustrated by the presence of large amounts of red blood cells in the muscle (Fig. 3B).

When BthTX-I was preincubated with rBaltMIP, a decrease in its local damage capacity could be noticed by the integrity of muscle fibers and the decreased number of leukocyte infiltrates (Fig. 3C).
Also, rBaltMIP greatly inhibited the myotoxic activity of BthTX-I when compared to the antivenom (Fig. 3E). When B. jararacussu crude venom was previously incubated with rBaltMIP, a higher preservation of muscle fibers and decreased leukocyte infiltrates could also be observed, but the red blood cells were still present (Fig. 3D). This hemorrhage was only inhibited by preincubation of crude venom with the commercial antivenom (Fig. 3F), while the preservation of muscle fibers was more evident after preincubation with rBaltMIP.

Trying to validate the hypothesis that rBaltMIP can complement the conventional antivenom, the inhibitor was added to the

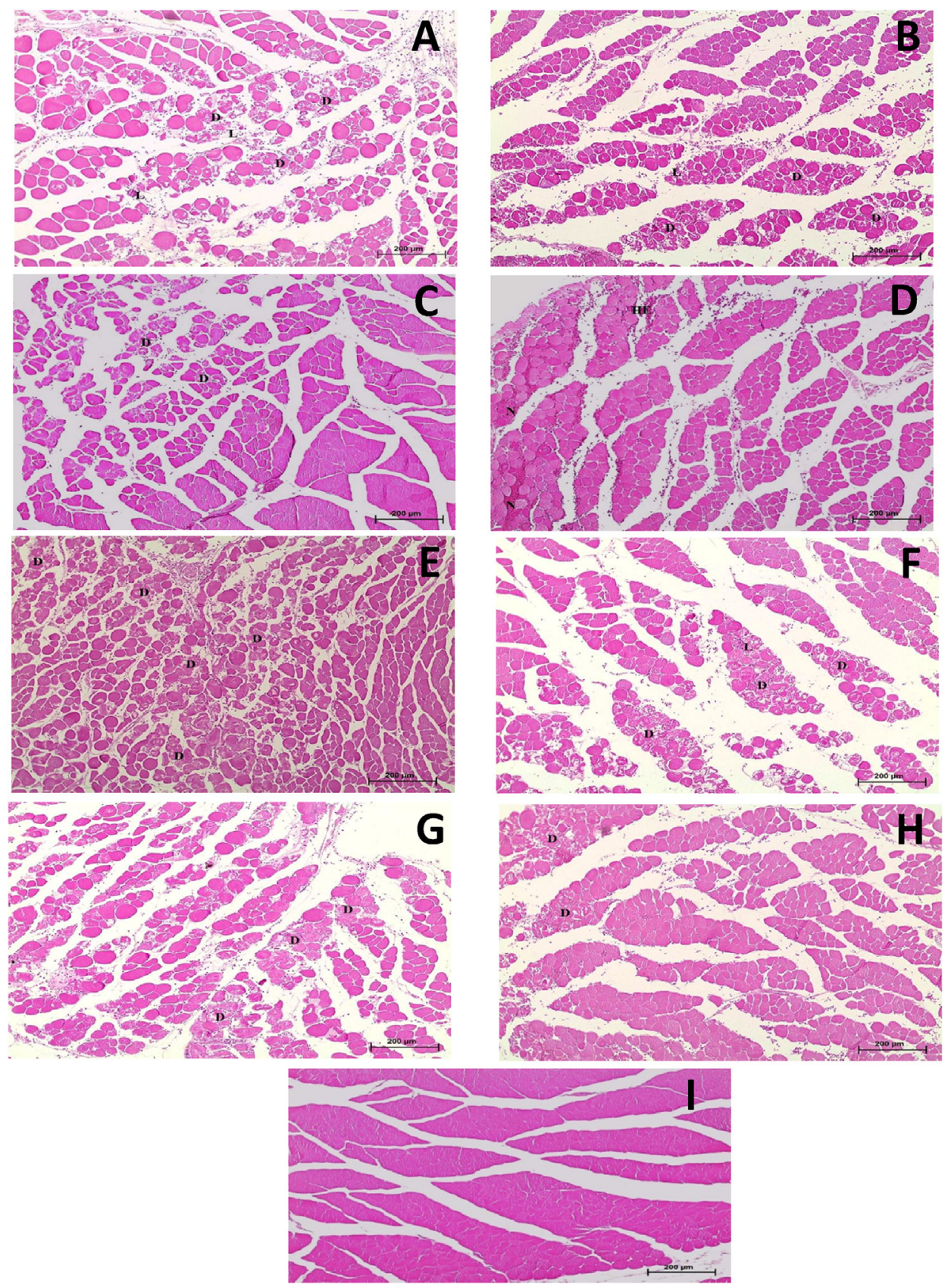

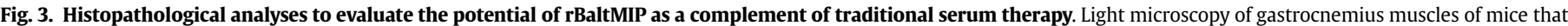

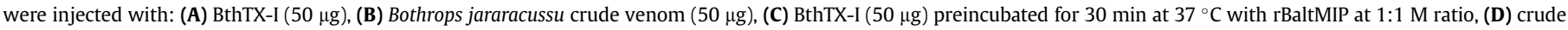

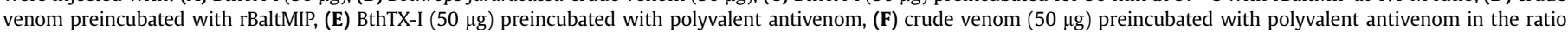

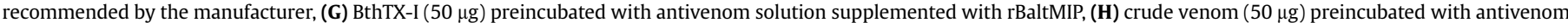

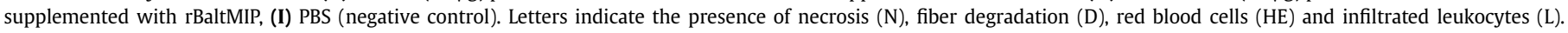
Bar $=200 \mu \mathrm{M}$. 

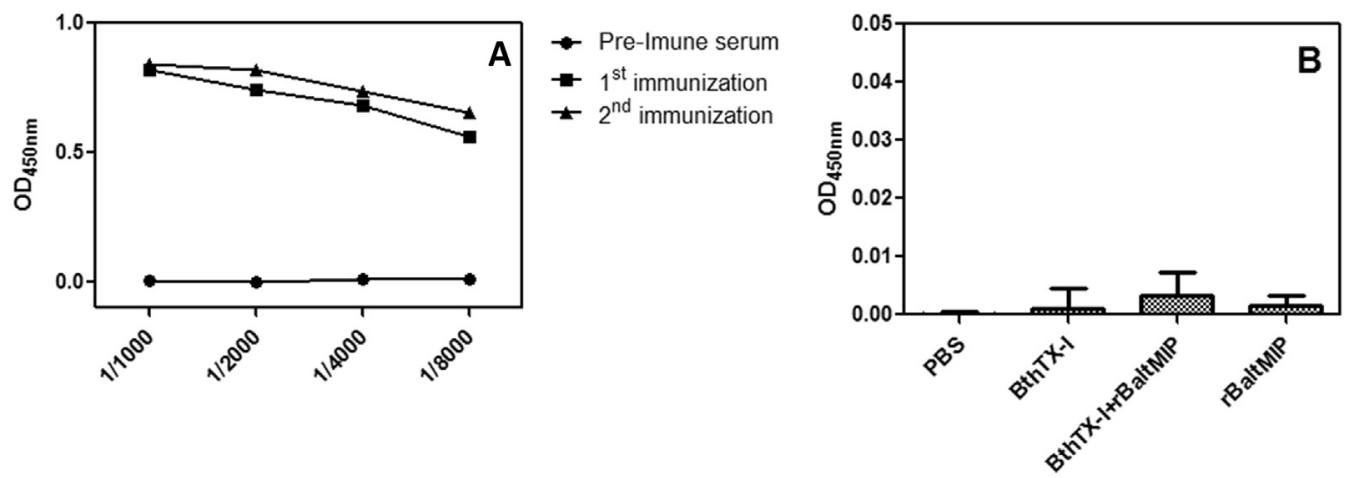

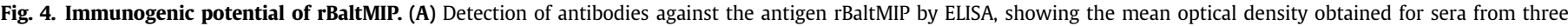

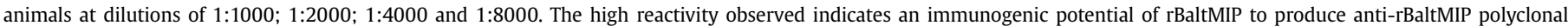

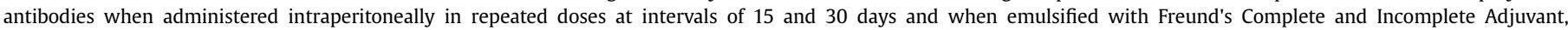

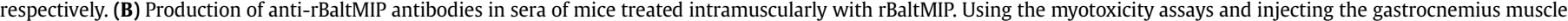

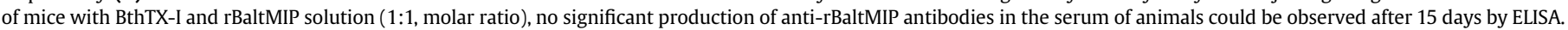

commercial antivenom and incubated with BthTX-I or B. jararacussu crude venom for $30 \mathrm{~min}$ prior to injection. Histopathology of muscle fibers that were inoculated with BthTX-I preincubated with the antivenom supplemented with rBaltMIP (Fig. 3G) showed that complemented antivenom solution was able to reduce muscle damage compared to BthTX-I, and especially to BthTX-I previously incubated only with antivenom. In addition, the crude venom preincubated with the antivenom supplemented with rBaltMIP (Fig. $3 \mathrm{H}$ ) induced less muscle damage in comparison to the crude venom alone or previously incubated only with the antivenom, and also induced less hemorrhage, suggesting an apparent synergistic effect.

The synergic effect of rBaltMIP with commercial antivenom was more evident when $B$. jararacussu crude venom was used. When the crude venom was injected in mice muscle, muscle fiber damage and intense hemorrhage could be observed (Fig. 3B), evidencing the local effects induced by myotoxins (among them Lys49 PLA Plike) $_{2}$ and also hemorrhage caused by other proteins (such as metalloproteases). This hemorrhage was inhibited after preincubation of crude venom with the commercial antivenom, although little preservation of the muscle fibers was observed (Fig. 3F). Other interesting observation could be made when rBaltMIP was preincubated with crude venom, since muscle fibers were preserved, but hemorrhagic activity was still present. However, when the crude venom was preincubated with the antivenom supplemented with rBaltMIP, an apparent synergic effect could be noted, since muscle damage (caused by myotoxins, which was specifically inhibited by rBalMIP) and hemorrhage (inhibited by antivenom) were both decreased (Fig. $3 \mathrm{H}$ ).

Several authors have suggested supplementation of antiophidian serum with natural inhibitors (Lizano et al., 2003; Oliveira et al., 2011; Santos-Filho et al., 2015a, 2014, 2011). Lizano et al. (Lizano et al., 2003) evaluated and confirmed the hypothesis that natural $\alpha$ PLIs could complement the conventional serum therapy. However, some questions were raised by these authors. The first one was about the limitation related to the availability of inhibitors, since they are isolated from snake blood, which are insufficient and ecologically unacceptable sources of inhibitors for commercial purposes. As mentioned earlier, the key to this problem would be the heterologous expression of proteins by molecular biology techniques, thus being a resource used to obtain greater quantities of active biomolecules. The second important question raised by Lizano et al. (Lizano et al., 2003) was about the toxicity and the immunogenic potential of $\alpha$ PLIs. According to them, after PLIs administration, an immune response would initiate, which could lead to anaphylactic reactions and inhibition of its therapeutic effects.

Considering this and aiming at the possible commercial use of plasma inhibitors in complementing conventional snake antivenoms, the immunogenic potential of rBaltMIP was evaluated in BALB/c mice using a conventional intraperitoneal immunization protocol in the presence of Freud's adjuvant. Polyclonal antibodies against rBaltMIP were detected at all tested serum dilutions $(1: 1000,1: 2000,1: 4000$ and $1: 8000)$ by ELISA, and, as expected, pre-immune sera did not present anti-rBaltMIP antibodies (Fig. 4A). These results suggest that rBaltMIP is immunogenic when administered intraperitoneally in repeated doses (0,15 and 30 days) in the presence of adjuvants. In the literature, there has been reported that a robust primary antibody response against proteins might occur even through a single injection without adjuvants (Gore et al., 2004). Interestingly, alarmins released by necrotic tissue, such as high-mobility group box 1 (HMGB1), are potent endogenous adjuvants that could enhance the primary antibody response to poorly immunogenic antigens (Rovere-Querini et al., 2004). Based on this information, we decided to check whether the immunogenic property of rBaltMIP could also be detected in the sample sera of mice that were used in the test of myotoxicity neutralization. For this, a myotoxicity inhibition test was conducted by injecting rBaltMIP with BthTX-I at 1:1 M ratio, and collecting and separating the animal's blood after 15 days, evaluating sera by ELISA for the production or not of anti-rBaltMIP antibodies. Interestingly, we did not detect the production of anti-rBaltMIP antibodies when the inhibitor was used as a treatment against the myotoxicity induced by BthTX-I (Fig. 4B). This result could be partially explained by experimental differences between those approaches: i) use of Freud's adjuvant or not; ii) distinct antigen doses; iii) number of antigens injections; iv) immunization route; v) tissue microenvironment. Nonetheless, further studies are needed to better understand the cellular and molecular mechanisms associated with the immunogenicity of this recombinant alpha-type myotoxin inhibitor, including the mapping of its functional site, in order to find peptides from this inhibitor with the capacity of neutralizing BthTX-I and -II with low immunogenicity, as described for another PLI (Thwin et al., 2002).

\section{Conclusion}

In conclusion, our studies showed important functional features for rBaltMIP, suggesting possible applications that could be useful not only for therapeutic purposes, but also in biotechnological 
industries studying the mechanisms of snake toxins, in the design of synthetic inhibitors and in the comprehension of other functional and structural characteristics of myotoxins. Moreover, considering that the anti-myotoxic effect of rBaltMIP is not associated with a primary antibody response against itself, it is possible to suggest its viable utilization as an important recombinant molecule to design additional antivenom therapy against Bothrops alternatus (Rhinocerophis alternatus) envenomation.

\section{Conflict of interest}

The authors declare that there is no conflict of interest.

\section{Ethical statement}

The authors Norival A. Santos-Filho, Tiago S. de Sousa, Johara Boldrini-França, Ludier K. Santos-Silva, Danilo L. Menaldo, Flávio Henrique-Silva, Adélia C.O. Cintra, Helen J. Laure, Carla C. N. Mamede, Fábio de Oliveira, Thalita B. Riul, Marcelo Dias-Baruffi, José C. Rosa and Suely V. Sampaio declare that manuscript named "rBaltMIP, a recombinant alpha-type myotoxin inhibitor from Bothrops alternatus snake, as a potential complement of antivenom therapy" complies with the Elsevier Ethical Guidelines for Journal Publication.

\section{Acknowledgments}

The authors gratefully acknowledge the financial support of São Paulo Research Foundation (FAPESP, grant \#2008/10760-4 and \#2011/23236-4), Coordination for the Improvement of Higher Education Personnel (CAPES) and the National Council for Scientific and Technological Development (CNPq grant \#467646/2014-7 and \#476932/2012-2). Also, the research leading to these results has received support and funding from the Núcleo de Apoio à Pesquisa em Doenças Inflamatórias (NAPDIN, grant \#11.1.21625.01.0). Additionally, authors would like to express their gratitude to João J. Franco and Luiz F. Tucci for the technical support.

\section{Transparency document}

Transparency document related to this article can be found online at http://dx.doi.org/10.1016/j.toxicon.2016.10.018.

\section{References}

Bonfim, V.L., de Carvalho, D.D., Ponce-Soto, L.A., Kassab, B.H., Marangoni, S., 2009 Toxicity of phospholipases A2 D49 (6-1 and 6-2) and K49 (Bj-VII) from Bothrops jararacussu venom. Cell Biol. Toxicol. 25, 523-532.

Chippaux, J.P., 2015. Epidemiology of envenomations by terrestrial venomous animals in Brazil based on case reporting: from obvious facts to contingencies. J. Venom. Anim. Toxins Incl. Trop. Dis. 21, 13

Cintra, A.C., Marangoni, S., Oliveira, B., Giglio, J.R., 1993. Bothropstoxin-I: amino acid sequence and function. J. Protein Chem. 12, 57-64.

Cruz, L.S., Vargas, R., Lopes, A.A., 2009. Snakebite envenomation and death in the developing world. Ethn. Dis. 19. S1-42-46.

Estevão-Costa, M.I., Fernandes, C.A., Mudadu, M.e.A., Franco, G.R., Fontes, M.R. Fortes-Dias, C.L., 2016. Structural and evolutionary insights into endogenous alpha-phospholipase $A_{2}$ inhibitors of latin American pit vipers. Toxicon 112, 35-44.

Faure, G., 2000. Natural inhibitors of toxic phospholipases $A_{2}$. Biochimie 82 , $833-840$.

Fortes-Dias, C.L., 2002. Endogenous inhibitors of snake venom phospholipases $A_{2}$ in the blood plasma of snakes. Toxicon 40, 481-484.

Fuly, A.L., Calil-Elias, S., Martinez, A.M., Melo, P.A., Guimarães, J.A., 2003. Myotoxicity induced by an acidic Asp-49 phospholipase $A_{2}$ isolated from Lachesis muta snake venom. Comparison with lysophosphatidylcholine. Int. J. Biochem. Cell Biol. 35, 1470-1481.

Fusco, L.S., Rodríguez, J.P., Teibler, P., Maruñak, S., Acosta, O., Leiva, L., 2015. New immunization protocol to produce crotalic antivenom combining Crotalus durissus terrificus venom and its PLA2. Biologicals 43, 62-70.

Gore, E.R., Gower, J., Kurali, E., Sui, J.L., Bynum, J., Ennulat, D., Herzyk, D.J., 2004
Primary antibody response to keyhole limpet hemocyanin in rat as a model for immunotoxicity evaluation. Toxicology 197, 23-35.

Gutiérrez, J.M., 2014. Current challenges for confronting the public health problem of snakebite envenoming in Central America. J. Venom. Anim. Toxins Incl. Trop. Dis. $20,7$.

Gutiérrez, J.M., León, G., Lomonte, B., 2003. Pharmacokinetic-pharmacodynamic relationships of immunoglobulin therapy for envenomation. Clin. Pharmacokinet. 42, 721-741.

Habib, A.G., 2013. Public health aspects of snakebite care in west Africa: perspectives from Nigeria. J. Venom. Anim. Toxins Incl. Trop. Dis. 19, 27.

Harlow, E., Lane, D., 1988. Antibodies: A Laboratory Manual. Cold Spring Harbor Laboratory, Cold Spring Harbor, NY, pp. 1-22.

Harrison, R.A., Hargreaves, A., Wagstaff, S.C., Faragher, B., Lalloo, D.G., 2009. Snake envenoming: a disease of poverty. PLoS Negl. Trop. Dis. 3, e569.

Hifumi, T., Sakai, A., Kondo, Y., Yamamoto, A., Morine, N., Ato, M., Shibayama, K., Umezawa, K., Kiriu, N., Kato, H., Koido, Y., Inoue, J., Kawakita, K., Kuroda, Y., 2015. Venomous snake bites: clinical diagnosis and treatment. J. Intensive Care 3,16 .

Homsi-Brandeburgo, M.I., Queiroz, L.S., Santo-Neto, H., Rodrigues-Simioni, L., Giglio, J.R., 1988. Fractionation of Bothrops jararacussu snake venom: partial chemical characterization and biological activity of bothropstoxin. Toxicon 26, 615-627.

Itzhaki, R.F., Gill, D.M., 1964. A micro-biuret method for estimating proteins. Anal. Biochem. 9, 401-410.

Kini, R.M., Evans, H.J., 1989. A model to explain the pharmacological effects of snake venom phospholipases $A_{2}$. Toxicon 27, 613-635.

Krifi, M.N., El Ayeb, M., Dellagi, K., 1999. The improvement and standardization of antivenom production in developing countries: comparing antivenom quality, therapeutical efficiency, and cost. J. Venom. Animals Toxins 5, 128-141.

Kulkeaw, K., Chaicumpa, W., Sakolvaree, Y., Tongtawe, P., Tapchaisri, P., 2007. Proteome and immunome of the venom of the Thai cobra, Naja kaouthia. Toxicon 49, 1026-1041.

Laemmli, U.K., Mölbert, E., Showe, M., Kellenberger, E., 1970. Form-determining function of the genes required for the assembly of the head of bacteriophage T4. J. Mol. Biol. 49, 99-113.

Landucci, E.C., Dias, Q.C., Marangoni, F.A., Vilca-Quispe, A., Valeriano-Zapana, J.A., Torres-Huaco, F.D., Martins-de-Souza, D., Marangoni, S., Ponce-Soto, L.A., 2012. Purification and inflammatory edema induced by two PLA 2 (Anch TX-I and Anch TX-II) from sea anemone Anthothoe chilensis (Actiniaria: sagartiidae). Comp. Biochem. Physiol. B Biochem. Mol. Biol. 161, 170-177.

Lizano, S., Angulo, Y. Lomonte, B., Fox, J.W., Lambeau, G., Lazdunski, M., Gutiérrez, J.M., 2000. Two phospholipase $A_{2}$ inhibitors from the plasma of Cerrophidion (Bothrops) godmani which selectively inhibit two different group-II phospholipase $\mathrm{A}_{2}$ myotoxins from its own venom: isolation, molecular cloning and biological properties. Biochem. J. 346 (3), 631-639.

Lizano, S., Domont, G., Perales, J., 2003. Natural phospholipase $\mathrm{A}_{2}$ myotoxin inhibitor proteins from snakes, mammals and plants. Toxicon 42, 963-977.

Lizano, S., Lomonte, B., Fox, J.W., Gutiérrez, J.M., 1997. Biochemical characterization and pharmacological properties of a phospholipase $A_{2}$ myotoxin inhibitor from the plasma of the snake Bothrops asper. Biochem. J. 326 (Pt 3), 853-859.

Lomonte, B., Angulo, Y., Calderón, L., 2003a. An overview of lysine-49 phospholipase $A_{2}$ myotoxins from crotalid snake venoms and their structural determinants of myotoxic action. Toxicon 42, 885-901.

Lomonte, B., Angulo, Y., Moreno, E., 2010. Synthetic peptides derived from the Cterminal region of Lys49 phospholipase $A_{2}$ homologues from viperidae snake venoms: biomimetic activities and potential applications. Curr. Pharm. Des. 16, 3224-3230.

Lomonte, B., Angulo, Y., Santamaría, C., 2003b. Comparative study of synthetic peptides corresponding to region 115-129 in Lys49 myotoxic phospholipases $A_{2}$ from snake venoms. Toxicon 42, 307-312.

Marcussi, S., Sant'Ana, C.D., Oliveira, C.Z., Rueda, A.Q., Menaldo, D.L., Beleboni, R.O., Stabeli, R.G., Giglio, J.R., Fontes, M.R., Soares, A.M., 2007. Snake venom phospholipase $A_{2}$ inhibitors: medicinal chemistry and therapeutic potential. Curr. Top. Med. Chem. 7, 743-756.

Morais, V.M., Massaldi, H., 2009. Snake antivenoms: adverse reactions and production technology. J. Venom. Animals Toxins Incl. Trop. Dis. 15, 2-18.

Nishida, M., Okamoto, M., Ohno, A., Okumura, K., Hayashi, K., Ikeda, K., Inoue, S., 2010. Inhibitory activities of the heterotrimers formed from two $\alpha$-type phospholipase $A_{2}$ inhibitory proteins with different enzyme affinities and importance of the intersubunit electrostatic interaction in trimer formation. Biochim. Biophys. Acta 1804, 2121-2127.

Nunes, D.C., Rodrigues, R.S., Lucena, M.N., Cologna, C.T., Oliveira, A.C. Hamaguchi, A., Homsi-Brandeburgo, M.I., Arantes, E.C., Teixeira, D.N., UeiraVieira, C., Rodrigues, V.M., 2011. Isolation and functional characterization of proinflammatory acidic phospholipase $A_{2}$ from Bothrops leucurus snake venom. Comp. Biochem. Physiol. C Toxicol. Pharmacol. 154, 226-233.

Núñez, C.E., Angulo, Y., Lomonte, B., 2001. Identification of the myotoxic site of the Lys49 phospholipase $\mathrm{A}_{2}$ from Agkistrodon piscivorus piscivorus snake venom: synthetic C-terminal peptides from Lys49, but not from Asp49 myotoxins, exert membrane-damaging activities. Toxicon 39, 1587-1594.

Okumura, K., Ohno, A., Nishida, M., Hayashi, K., Ikeda, K., Inoue, S., 2005. Mapping the region of the alpha-type phospholipase $A_{2}$ inhibitor responsible for its inhibitory activity. J. Biol. Chem. 280, 37651-37659.

Oliveira, C.Z., Menaldo, D.L., Marcussi, S., Santos-Filho, N.A., Silveira, L.B., BoldriniFranca, J., Rodrigues, V.M., Soares, A.M., 2008. An alpha-type phospholipase A2 
inhibitor from Bothrops jararacussu snake plasma: structural and functional characterization. Biochimie 90, 1506-1514.

Oliveira, C.Z., Santos-Filho, N.A., Menaldo, D.L., Boldrini-Franca, J., Giglio, J.R., Calderon, L.A., Stabeli, R.G., Rodrigues, F.H.S., Tasic, L., da Silva, S.L., Soares, A.M., 2011. Structural and functional characterization of gamma-type phospholipase $\mathrm{A}_{2}$ inhibitor from Bothrops jararacussu snake plasma. Curr. Top. Med. Chem. 11, 2509-2519.

Prado, N.D., Pereira, S.S., da Silva, M.P., Morais, M.S., Kayano, A.M., Moreira-Dill, L.S., Luiz, M.B., Zanchi, F.B., Fuly, A.L., Huacca, M.E.F., Fernandes, C.F., Calderon, L.A. Zuliani, J.P., Pereira da Silva, L.H., Soares, A.M., Stabeli, R.G., Fernandes, C.F.C., 2016. Inhibition of the myotoxicity induced by Bothrops jararacussu venom and isolated phospholipases $\mathrm{A}_{2}$ by specific camelid single-domain antibody fragments. PLoS One 11, e0151363.

Quirós, S., Alape-Girón, A., Angulo, Y., Lomonte, B., 2007. Isolation, characterization and molecular cloning of AnMIP, a new alpha-type phospholipase $\mathrm{A}_{2}$ myotoxin inhibitor from the plasma of the snake Atropoides nummifer (Viperidae: crotalinae). Comp. Biochem. Physiol. B Biochem. Mol. Biol. 146, 60-68.

Rovere-Querini, P., Capobianco, A., Scaffidi, P., Valentinis, B., Catalanotti, F., Giazzon, M., Dumitriu, I.E., Müller, S., Iannacone, M., Traversari, C., Bianchi, M.E. Manfredi, A.A., 2004. HMGB1 is an endogenous immune adjuvant released by necrotic cells. EMBO Rep. 5, 825-830.

Santos-Filho, N., Silveira, L., Boldrini-França, J., 2015a. Myotoxin inhibitors, Toxins and drug discovery. Springer, Netherlands, pp. 1-24.

Santos-Filho, N.A., Boldrini-França, J., Santos-Silva, L.K., Menaldo, D.L., HenriqueSilva, F., Sousa, T.S., Cintra, A.C., Mamede, C.C., Oliveira, F., Arantes, E.C., Greggi Antunes, L.M., Cilli, E.M., Sampaio, S.V., 2014. Heterologous expression and biochemical and functional characterization of a recombinant alpha-type myotoxin inhibitor from Bothrops alternatus snake. Biochimie 105, 119-128.

Santos-Filho, N.A., Fernandes, C.A.H., Menaldo, D.L., Magro, A.J., Fortes-Dias, C.L., Estevao-Costa, M.I., Fontes, M.R.M., Santos, C.R., Murakami, M.T., Soares, A.M., 2011. Molecular cloning and biochemical characterization of a myotoxin inhibitor from Bothrops alternatus snake plasma. Biochimie 93, 583-592.

Santos-Filho, N.A., Lorenzon, E.N., Ramos, M.A., Santos, C.T., Piccoli, J.P., Bauab, T.M., Fusco-Almeida, A.M., Cilli, E.M., 2015b. Synthesis and characterization of an antibacterial and non-toxic dimeric peptide derived from the C-terminal region of Bothropstoxin-I. Toxicon 103, 160-168.

Santos-Filho, N.A., Silveira, L.B., Oliveira, C.Z., Bernardes, C.P., Menaldo, D.L., Fuly, A.L., Arantes, E.C., Sampaio, S.V., Mamede, C.C.N., Beletti, M.E., de
Oliveira, F., Soares, A.M., 2008. A new acidic myotoxic, anti-platelet and prostaglandin I-2 inductor phospholipase $\mathrm{A}_{2}$ isolated from Bothrops moojeni snake venom. Toxicon 52, 908-917.

Scheske, L., Ruitenberg, J., Bissumbhar, B., 2015. Needs and availability of snake antivenoms: relevance and application of international guidelines. Int. J. Health Policy Manag. 4, 447-457.

Scopes, R.K., 1974. Measurement of protein by spectrophotometry at $205 \mathrm{~nm}$. Anal. Biochem. 59, 277-282.

Soares, A.M., Giglio, J.R., 2003. Chemical modifications of phospholipases A from snake venoms: effects on catalytic and pharmacological properties. Toxicon 42 , $855-868$.

Soares, A.M., Marcussi, S., Stábeli, R.G., França, S.C., Giglio, J.R., Ward, R.J. Arantes, E.C., 2003. Structural and functional analysis of BmjMIP, a phospholipase $A_{2}$ myotoxin inhibitor protein from Bothrops moojeni snake plasma. Biochem. Biophys. Res. Commun. 302, 193-200.

Soares, A.M., Sestito, W.P., Marcussi, S., Stábeli, R.G., Andrião-Escarso, S.H., Cunha, O.A., Vieira, C.A., Giglio, J.R., 2004. Alkylation of myotoxic phospholipases $A_{2}$ in Bothrops moojeni venom: a promising approach to an enhanced antivenom production. Int. J. Biochem. Cell Biol. 36, 258-270.

Thwin, M.M., Gopalakrishnakone, P., Kini, R.M., Armugam, A., Jeyaseelan, K., 2000 Recombinant antitoxic and antiinflammatory factor from the nonvenomous snake Python reticulatus: phospholipase $\mathrm{A}_{2}$ inhibition and venom neutralizing potential. Biochemistry 39, 9604-9611.

Thwin, M.M., Satish, R.L., Chan, S.T., Gopalakrishnakone, P., 2002. Functional site of endogenous phospholipase $A_{2}$ inhibitor from python serum. Eur. J. Biochem. 269, 719-727.

Vesterberg, O., 1972. Isoelectric focusing of proteins in polyacrylamide gels. Biochim. Biophys. Acta 257, 11-19.

WHO, 2012. WHO expert committee on biological standardization. World Health Organ Tech. Rep. Ser. 1-228 back cover.

Zolfagharian, H., Dounighi, N.M., 2015. Study on development of Vipera lebetina snake anti-venom in chicken egg yolk for passive immunization. Hum. Vaccin Immunother. 11, 2734-2739.

Zuliani, J.P. Fernandes, C.M., Zamuner, S.R., Gutiérrez, J.M. Teixeira, C.F. 2005. Inflammatory events induced by Lys -49 and Asp- 49 phospholipases $A_{2}$ isolated from Bothrops asper snake venom: role of catalytic activity. Toxicon 45 $335-346$. 